\title{
NOTES SUR LA GENÈSE DU CONCEPT DE « MONUMENTALITÉ »
}

Révulsive fut la découverte des Amériques : les idées européennes y prirent plus d'un pli ; ainsi du concept d'humanité que réformèrent les théologiens de Salamanque à la faveur de la querelle des Indes. Plus tard, sur une matière moins relevée, mais sensible, on verra les conditions de la monumentalité urbaine reconsidérées, au retour de voyageurs que stupéfient New York et Chicago. En Europe, fortifiée par des superstitions tenaces, l'image de la monumentalité reposait, de longue date, sur l'incorporation de sites sacralisés dans des réseaux de formes symboliques ${ }^{1}$. Or, dès le dernier tiers du XIX' siècle, les touristes éveillés rapportent de leurs croisières outre-Atlantique le souvenir de schèmes inverses. C'est, par exemple, Jules Leclercq qui publie, en 1877, que New York a « des constructions énormes, des hôtels somptueux, d'immenses magasins $[\ldots]$ mais point de vrais monuments ${ }^{2}$. Les disparates d'échelle et de fonctions surprennent d'autant plus le visiteur attentif que la question de la monumentalité privée, rivale de la publique, commence alors de se poser avec insistance dans les capitales européennes, avant même que le terme de "monumentalité " fût en usage. Les pages qui suivent visent à expliciter le passage de l'idée de monument à la notion

1. On peut dire que la théorie de ce mode d'organisation édilitaire est achevée par les commentaires des « urbanistes » de la Révolution française et leurs épigones. Voir, p. ex., l'article de Pierre-Jean-Baptiste CHAUSSARD, « Monumens de l'Héroïsme Français », qui a pour sous-titre : « nécessité de ramener à un plan unique, et de coordonner à ceux déjà existans, les monumens qu'on propose d'élever à Paris... ", La Décade philosophique, littéraire et politique, 30 pluviôse, an IX, p. 361 sq.

2. Jules LeCLercQ, Un Eté en Amérique..., Paris, 1877, p. 33. Le propos ne fait qu'amplifier les remarques de Chateaubriand publiées un demi-siècle plús tôt, avant que ne s'érigent de colossaux édifices utilitaires.

Revue de synthèse: IVe $\mathrm{S} . \mathrm{N}^{\circ} 1$, janv.-mars 1987. 
de monumentalité, étant entendu que chacun de ces termes revêt de multiples significations qui continuent de s'entregloser.

\section{UN CAS D'ANTHOLOGIE}

Les candidats au baccalauréat des années 1920 pouvaient se frotter d'histoire dans les 1184 pages du Malet ${ }^{3}$, monument d'instruction républicaine dont les derniers mots disent la justesse de ses préjugés ${ }^{4}$. L'ouvrage était discrètement illustré de « gravures d'après les documents »; l'une d'elles est remarquable en ce qu'elle manifeste l'essence de l'idée de monument, réduite à ses attributs indiscutables, hors du caractère de monumentalité. La légende commente l'image d'un « hideux monument connu sous le nom de Tour des Crânes », proche de Nich, daté 1809 : « Non loin de là, un millier de Serbes occupaient la redoute de Kamenitsa. Assiégés par les Turcs, ils se firent sauter au moment où l'ennemi " y pénétra. " Les Turcs décapitèrent tous les cadavres [...] ils édifièrent une tour dont le revêtement était entièrement fait des têtes des vaincus. " Les Serbes reprirent Nich en 1878 ; « la tour était encore intacte [...] Ils enlevèrent alors les crânes, les déposèrent dans un ossuaire et construisirent un abri grossier [...] Ce monument dit éloquemment la sauvagerie des Turcs $"$ " 5 . Les thèmes contraires de la colonne d'infamie et de la chapelle expiatoire se fondent ainsi dans le contour alvéolaire, maudit puis bénit. Si la fonction d'un monument est de remémorer à des fins d'édification, nul édifice n'approche davantage de son objet, ayant été pétri d'exactes reliques. Mais si l'on prétend que le rappel incorpore des signes d'idéalisation esthétique, la tour de Nich n'est alors rien de plus qu'un macabre truc de Turcs qu'une fureur sacrificielle de Serbes exacerba. Cet édifice suscite du malaise chez quiconque n'est pas intéressé à des motifs de piété patriotique. Le malaise tient sans doute à ce que ce monument n'est qu'un reliquaire ; il illustre trop bien dans l'ordre édiculaire l'obscénité de l'intime que les diaristes érigent en œuvre, avec pour seule excuse l'obsession de l'authentique. Le monument se distingue précisément du simple reliquaire par un codage esthétique

3. Plus précisément : $X I X^{e}$ siecle, histoire contemporaine (1815-1920), par A. MALET, P. Grillet, J. IsAac, Paris, Hachette, s.d.

4. Ibid., p. 1176 : "...la République allemande, établie par accident, ne semble avoir encore qu'une existence précaire. Mais sa consolidation paraît indispensable au maintien de la paix dans le monde. "

5. Ibid., p. 412. Une tour analogue est signalée dans Encyclopediana, Paris, 1791, p. 701, formée des os d'Espagnols défaits par les Infidèles en 1560, dans l'île de Gerbe (Djerba). 
interposé qui en fonde la généalogie : trace de mémorable, ancrée dans la chronique, sujette à l'érosion du temps, le monument, dans son acception traditionnelle est un artifice qui "parle " et vieillit tout ensemble, c'est-à-dire un artifice que la durée voue à la méconnaissance, à l'oubli, au mythe.

\section{DÉFINITIONS ET VARIÉTÉS}

À l'époque dite classique, les définitions du terme "monument " avouent des rapports antagoniques avec la temporalité. Furetière privilégie le regard vers le passé, l'Académie pense l'avenir d'une destination. Selon la Compagnie, le monument est « marque publique qu'on laisse à la postérité pour conserver la mémoire de quelque personne illustre, ou de quelque action célèbre $"{ }^{6}$ : le Dictionnaire universel retient le " témoignage qui nous reste de quelque grande puissance ou grandeur des siècles passez $"{ }^{7}$. Entre les deux sentences joue la diversité de la lecture à l'écriture ; toutes deux supposent du grand et du mémorable, mais la dernière incorpore l'effacement physique de l'intention qui a motivé l'érection monumentale.

L'oubli de la dédicace est un malheur contre lequel l'imagination s'est exercée avec des succès divers. L'idée d'un monument inaltérable fut formée en 1787 par un astronome courtisan qui proposa d'attribuer le nom de Frédéric à des étoiles inconnues; en 1792, Chodowiecki put figurer l'extase du Prince contemplant la nouvelle constellation de la "Gloire de Frédéric " ${ }^{8}$. Par la suite, l'invention d'éléments chimiques suscitera des attributions analogues, à la manière de la désignation de territoires inconnus par les explorateurs. Ces monuments supposés inaltérables sont encore bien imparfaits si on les compare aux monuments idéals dont on déclare que le motif transcende tout essai de célébration. De sa nature, le monument idéal est pur effet de rhétorique ; soit, en exemple, un exorde de Champagny : « L'Empereur dont la gloire est impérissable et doit survivre à tous les monumens destinés à en perpé-

6. Dictionnaire de l'Académie francoise, Paris, 1694, $\mathrm{V}^{\circ}$, "Monument ». Le Grand vocabulaire françois en son t. XVIII (Paris, 1771) retient une définition similaire : « Marque publique pour transmettre à la postérité la mémoire de quelque personne illustre, ou de quelque action célèbre, de quelque événement mémorable. " Dans les deux références, le caractère public est exclusif. La $7^{e}$ éd. de l'Académie admettra en 1879 le cáractère particulier.

7. Dictionnaire universel..., Paris, $1690, V^{\circ}$, « Monument ».

8. Herbert SchraDE, Das deutsche Nationaldenkmal, Munich, 1934, p. 71. 
tuer le souvenir... ${ }^{9}$; ainsi, faut-il supposer un monument incorporel, cause de monuments concrets subalternes; il en résulte l'obligation d'identifier la contingence du prototype à la révélation de l'idée d'Empire...

Résumé dans sa dédicace, le monument idéal a pour contrepartie le monument impossible, savoir le monument sans dédicace, ou encore celui qui a pour dédicataire un héros inconnu qu'il faut distinguer du "Tombeau du Soldat inconnu » dont les restes ont une existence empirique. Mais la collection des variétés monumentales serait incomplète sans la paradoxale, celle qu'illustre Le Corbusier s'extasiant sur des monuments non monumentaux : « un banc » qui a « sur le sable [...] une place définitive » ou « un figuier » contemporain d'une maison, «monument [en] sa place indiscutable ${ }^{10}$. $\grave{A}$ ce parti pris se rattache une définition idiopathique : «Nous nommons monumental ce qui contient des formes pures assemblées suivant une loi harmonieuse " ${ }^{11}$. Par le ton, le propos se veut inaugural, ce qui n'est pas pour étonner de la part de son auteur. Mais a-t-on remarqué qu'il n'a pu être énoncé et entendu possible qu'en vertu de la perte d'intention typique des monuments, au sens qu'assurait jadis leur étymologie ? Il est également significatif que l'apparition tardive de l'épithète monumental( $e$ ) dans les langues de culture ${ }^{12}$ coïncide avec l'altération et la dispersion du sens originaire de monument. La rupture est consommée, en 1771 , lorsque J.-F. Blondel qualifie de monument « tout édifice remarquable ». Le moment légitime l'assignation du caractère monumental et la recherche des effets qui y conviennent ${ }^{13}$; la vue s'éclaire désormais sur les

9. Circulaire du ministre de l'Intérieur, en date du 20 déc. 1806, repr. in Journal des arts, des sciences, de littérature et de politique, $\mathrm{n}^{\circ} 441, \mathrm{p} .4$. L'exorde introduit en fait à la mise au concours d'un " monument " qui " sera le plus auguste, le plus imposant de tous ceux que sa vaste imagination a conçus, et que son activité prodigieuse sait faire exécuter... » (ibid.) ; en l'occurrence, la puissance se délègue pour inspirer sa célébration. La supériorité des principes sur leurs signes est invoquée encore par $M^{\text {me }}$ DE STAEL en 1816 dans son article De l'esprit des traductions, in O.c., Paris, 1818, XVII, p. 398, où elle rappelle que "l'on se passe plutôt de tableaux et de monumens que des sentimens auxquels ils doivent être consacrés ».

10. Le Corbusier, Une Maison-Un Palais, Paris, 1928, p. 50.

11. Ibid., p. 52.

12. P. ex., l'éd. italienne du Dictionnaire de l'architecture d'Antoine-Chrysostôme QuATREMERE DE QUINCY, Mantoue, 1842, justifie « per molte ragioni " l'introduction de la " parola monumentale [...] in un dizionario d'architettura "; la dérivation française est expressément notée. Les premiers exemples que donne la Crusca sont tirés de Gioberti, etc.

13. L'article "Monumentale » de l'édition italienne citée de Quatremère s'achève par l'indication des principes qui doivent régler la production de "grandiosi effetti »; ils tiennent pour lui à la mise en cuvre de matériaux « solidi e d'una grande dimensione " propres à élever des " moli imponenti »; il loue expressément la " semplicità di mezzi " en l'opposant aux " combinazioni artificiali della scienza "; l'avis n'était pas universellement reçu. 
ressorts déterminant les signes esthétiques et les enjeux sociaux de ce qui n'est pas encore désigné du nom de monumentalité ${ }^{14}$.

En France, à l'époque révolutionnaire, la naturalisation du monumental se manifeste par la coexistence et la compétition de deux régimes distincts de projets d'architecture dont l'image et le texte témoignent. Deux régimes selon deux intentions ; l'une vise à en imposer par l'ampleur des volumes configurés et la variété des effets qui en résultent ; l'autre surenchérit sur la fonction édifiante des monuments en les prenant expressément pour support des propagandes ${ }^{15}$. La surenchère se renforçait, en outre, de ce que les intentions didactiques appelaient l'exécration corollaire des " signes de féodalité », ce qui était une manière auxiliaire de fonder une valeur de mémoire ${ }^{16}$. Rétrospectivement, les facteurs invoqués pour expliquer l'avènement d'une " pensée monumentale » ${ }^{17}$ peuvent

14. En 1959, le Dictionnaire alphabétique et analogique de la langue française de Paul ROBERT ignore «Monumentalité », mais son Supplément de 1972 l'accueille toutefois, renvoyant au Larousse mensuel en 1909, et indique, sans autre précision, une première occurrence en 1845 .

15. Dans les premiers temps de la Révolution, Gatreaux publie un Projet de monument, s.l.n.d., destiné à « mettre sous les yeux d'un Peuple immense les loix auxquelles il est soumis ; [...] par des architectures inaltérables, ]'homme connoitra ses droits [...] il apprendra ses devoirs et ce qui lui est imposé par l'ordre social et le bonheur de tous ", p. 3. C'était là un lieu commun ; il n'a pas attendu la Révolution pour se faire jour : le programme de l'utopique Olbie ou Essai sur les moyens de réformer les mours d'une nation de Jean-Baptiste SAY, Paris, an VIII, p. 77, multipliant « les murs qui parlent au peuple », est déjà développé dans un libelle publié à Stockholm en 1775, par le cryptonyme P.F.J.H. ; ne prétendait-il pas que tous les passages fussent couverts de " monumens publics propres à faire ressouvenir des leçons [...] reçues dans les écoles »? in De l'instruction publique, p. 112. Mais il faut distinguer dans " la muette éloquence des monumens ", l'affichage proprement dit de la communication symbolique. Le premier mode est illustré par l'abbé GREGOIRE; il prescrit le 21 nivôse an II que seul l' « idiome national " fleurisse sur les monuments ; la généralisation symbolisante est développée par Quatremère de Quincy lorsqu'il assimile les monuments aux « caractères d'une sorte de langue universelle, qui devient l'expression la plus éloquente des sensations et des affections publiques, surtout quand les gouvernemens savent la parler " ; cf. Notice historique sur [...] Gondoin, lue le 6 octobre 1821. La méfiance à l'égard d'un pouvoir ignorant se résout chez Karl Friedrich Wiebeking par le voeu insistant d'éduquer « les hommes qui tiennent les rênes des gouvernements »; cf. K. F. WIEBEKING, Analyse descriptive, historique et critique des monumens..., Munich, 1838 , t. II, p. 174. Le propos semble faire écho aux animadversions de J.-B. SAY qui souhaitait au tome V de son Cours complet d'économie politique pratique, Paris, 1829 , p. 220 , n., que les « hommes d'État » aient « du goût, en même temps que de l'économie et un jugement sain »; mais c'était dans son opinion, en vue de brider l'imagination des architectes, et de soumettre à « révision » leurs plans.

16. On ne peut désormais parler des différentes « valeurs » que supportent les monuments, valeurs d'art, d'ancienneté, historique, commémorative, etc. sans se référer aux pages denses qu'Alois RIEGL leur a consacrées dans Le Culte moderne des monuments, soudain accessible en France par deux traductions inégales - la meilleure étant publiée dans la coll. « In extenso ", Paris, 1985.

17. L'expression peut être relevée chez Louis-Pierre BaLTARD, dans son Introduction au Cours de théorie de l'année 1839, Paris, s.d., p. 18. Il serait intéressant de rechercher les occurrences antérieures du syntagme. L'idée, assurément, courait de longue date, ingrédient presque obligé de l'éloquence académique ; cf., p. ex., le passage intitulé « Magnificenza e disegno ", in F. S. BrunETTI, Trattenimenti scientifici..., Rome, 1755, p. 125. 
paraître bien fragiles : les contradictions idéologiques ont miné les intentions didactiques ; quant à la " grandiosité " des projets que l'on désignera par la suite de « néoclassiques », elle demeura affaire d'un petit nombre de dessinateurs exaltés, au mieux un thème de débats académiques ${ }^{18}$. Force est d'admettre, aujourd'hui, que l'avènement de la catégorie esthétique du monumental doive être compris de multiples points de vue et rallie des approches complémentaires ou contradictoires. L'histoire philologique est, en l'occurrence, un instrument de première nécessité, quoique imparfait. À ma connaissance, la dérivation adjective de monument en monumental - il y eut d'autres désinences, mais vite désuètes ${ }^{19}$ - s'enracine avec la vogue dont jouissent les Descriptions, puis les Statistiques " monumentales »; le premier genre fut inauguré par les travaux de l'Institut d'Égypte, le faste de leur présentation s'accordant, à point nommé, à la faveur légendaire qui émanait du paradigme égyptien ${ }^{20}$. L'archéologie qui aspirait depuis un demi-siècle à une dignité scientifique propre trouva en cette rencontre l'occasion de démontrer ses titres. Le monument d'érudition qu'elle produisit a publicisé, avant la lettre, la notion diffuse de patrimoine archéologique. De plus, il a contribué, quoique à un moindre degré, à fournir d'arguments, diversement entendus, le débat sur les attributs esthétiques de la monumentalité qui s'ouvrit, pour ne point se clore, au début du XIXc siècle, bien avant l'apparition du terme.

\section{MODES D'EXISTENCE DU MONUMENT}

Comme tout artifice humain, le monument peut être expliqué par une composition de propriétés antagoniques. La méthode a fait ses preuves et peut encore servir, même en un temps qui érige la complexité en cause. Aussi voudra-t-on bien admettre que la combinaison de couples de

18. « Grandiosité » est une manière recherchée de dire « grand style » ; on la trouve dans Raphaël Mengs. Le Traité de l'éducation, Paris, an III, de Jean-Gervais LA BENNE, vante « la grandiosité qu'on admire encore dans les chefs d'œuvre antiques » (p. 207). Mais le mot n'a pas connu de fortune durable. Au demeurant, lorsque G.-L. LE Camus substantifie l'adjectif correspondant, il le hasarde en italique, dans sa Lettre au $C^{e n}$. $G^{x a x}$, Paris, frimaire an IX, p. 10 : « la majesté et le grandiose de ce monument doivent se composer..." "

19. Émile LITTRÉ, qui ignore « monumentalité », procure toutefois « monumentaliser » et « monumenté "!

20. Dans son Essai [...] sur [...J Monge, Paris, 1819, Pierre-Charles-François DuPIN ne peut mieux louer la « description géodésique et monumentale de l'Égypte " qu'en la donnant comme une « entreprise vraiment polytechnique " (op. cit., p. 65) ; dans le feu de son enthousiasme, il propose que l'on borde l'axe des Tuileries, préalablement nivelé, d'une décoration de sphinx et d'obélisques monolithes (op. cit., p. 124). 
qualités opposées permette de dessiner des causalités, de fixer des systèmes de références, de déterminer, en définitive, plusieurs modes d'existence du monument.

Si on considère, par exemple, la fonction signalétique, c'est l'opposition de l'immédiat au récurrent, catégories de la temporalité qui lui donnera sa consistance, du double point de vue ontologique et empirique. $\mathrm{Si}$ on regarde le monument comme machine de pouvoir, la question se pose de décider entre actuel et simulé, ce qui revient à juger de l'adéquation des moyens à une entreprise de séduction. Regardé, enfin, comme document patrimonial, le monument rappelle la distinction du public et du privé, partant la virtualité de l'affaiblir dans la légitimation de moyens termes. Et l'on pourrait, sans doute, continuer l'énumération des couples antagonistes qui participent à la détermination de l'idée de monument, dans ses divers modes d'existence. Il est clair que la notion de monumental emprunte à ces divers modes, partant qu'elle dépasse en complexité sémantique la simple dérivation adjective complaisamment recueillie par les dictionnaires, qui ne font que signaler des usages indistinctement justifiés ${ }^{21}$. On supposera néanmoins que l'apparition de cette épithète - par la suite substantivée - fut nécessaire, de quelque manière qu'on l'entende. Par effet de mode, à tout le moins, ou en vertu d'une qualification esthétique raisonnée ; aucun des motifs n'excluant l'autre.

Sur le fait de la mode, les traces les plus discrètes décident. Revenue à Paris, après les fureurs révolutionnaires, $\mathbf{M}^{\mathrm{me}}$ de Genlis s'étonne que l'on dise alors « mon château " ou " mon hôtel ", pour " ma maison ", selon la coutume précédente. Le trait est anecdotique ; mais il révèle assez bien un goût d'ostentation et une façon de parvenir, mille fois attestés d'ailleurs, qui peuvent donner à penser que la venue de l'épithète "monumental " serait l'une des expressions d'un désir diffus de monument, dans une époque trouble où la force des appétits individuels se compose avec les rhétoriques de l'héroïsme. Mais cette vue superficielle ne contrevient aucunement à la recherche de traits constitutifs de l'esthétique du monumental que rend difficile, il est vrai, la mobilité d'un objet aussi peu saisissable que la mode et le goût qui lui sont relatifs.

Invoquer le goût donne matière à stigmatiser une composante problématique de la qualité monumentale, celle qui touche à la magnificence

21. Selon la $6^{e}$ éd. du Dictionnaire de Gattel, Paris, 1841 , « Monumental » se dit de ce «Qui a rapport aux monuments, qui est de la nature des monuments »; la $7^{\mathrm{e}}$ édition de l'Académie, en 1879, reprend, terme à terme, cette définition. Peu avant, E. Littré ne faisait qu'énoncer «Qui a rapport aux monuments »; mais, en 1887, Bescherelle procurera l'extension "Grandiose, durable..." ". 
en tant qu'elle illustre le pouvoir et que ses degrés rendent compte de la distinction des rangs dans le corps social. Les anciens auteurs séparaient avec méthode les moyens de produire l'effet de magnificence de la juste expression de cette magnificence qui est affaire de convenance ${ }^{22}$. Ici le jugement hésite : s'il est clair que grandeur des édifices et luxe des matériaux contribuent à cette expression, maint raisonneur a pu faire valoir une conception puritaine des aspects en signe de distinction ou en marque d'intelligence pratique ${ }^{23}$. Ces choses sont connues; elles le seraient davantage encore si l'on parvenait à se représenter correctement le jeu des rivalités symboliques et positives qu'exercent, au XIX' siècle, les acteurs de la commande architecturale, les auteurs de projets et les commentateurs des passions urbaines.

De sa lointaine constitution, l'idée de monument conserve cette propriété qu'il signifie, en un site, l'émanation d'un pouvoir. Autrement dit, le sens affiché, sacré ou profane n'importe, suppose le pouvoir qui l'autorise ; le monument n'est pleinement tel que dans la mesure où il est reconnu sur un registre sacramentel, identifié comme signe, délégation et manifeste du pouvoir ${ }^{24}$; c'est la raison pourquoi les vrais monuments attirent le vandalisme, sur le mode profanatoire ${ }^{25}$. À cet égard, c'est moins le caractère imposant de l'édifice qui le constitue

22. Cf., p. ex., Jacques BLoNDEl qui se réjouit dans l'Architecture françoise, Paris, 1752, t. I, p. 21, que l'on recherche désormais « la noblesse, la grandeur et la proportion relativement à l'esprit de convenance ". La convenance s'étend à la fois du point de vue de la commodité d'usage et de celui de la juste expression du decorum, selon les rangs. C'est en ce sens que MAILliER dans son Architecture, Paris, 1781, p. 5, légitime (en vers) les distinctions stylistiques : « Les biens, l'autorité, la valeur, la naissance, / Des Princes aux Sujets marquant la différence, / Exigèrent qu'on mît, pour distinguer les rangs, / Un style convenable à tous les bâtiments. " Le contre-type de cette norme est exprimé par Rigoley DE JuVignY, dans son essai De la décadence des lettres et des mours..., Paris, 2e éd., 1787 ; il đéplorait de « voir à côté des monumens immortelş du Siècle de Louis XIV, cette multitude de bâtimens \& d'ornemens qui n'appartiennent qu'aux Temples, aux Palais de nos Rois \& aux Édifices publics » (op. cit., p. 451).

23. Dans son Cours..., op. cit. supra n. 15, p. 221-222, J.-B. SAY affirme que « le public est en droit d'exiger [...] qu'on ne rende pas un édifice public plus fastueux que son objet ne le demande »; évoquant le projet d'une Bourse de commerce, il en décrit l'architecture, « noble, sévère, analogue à son objet [...] sans un luxe de colonnes qui en fît un temple plus vaste que n'en eurent jamais l'Italie ou la Grèce antique... ". Dans cette veine, relire Jean-Nicolas-Louis Durand, Giuseppe Antonio Borgnis, Louis Bruyère...

24. Cf., p. ex., ce propos d'A. QuATREMĖRE DE QuINCY tiré de son Rapport sur l'édifice dit de Sainte-Geneviève, Paris, 1791, p. 29 : «Votre édifice est un catéchisme figuré des devoirs de l'homme en société... "

25. Dans sa Lettre [...] sur le concours pour le Monument consacré à perpétuer les Noms des Défenseurs de la Patrie..., Paris, an IX, p. 11, G.-L. LE Camus expose " une considération majeure " qui naît du « désir de conservation qu'inspire un semblable monument ; c'est que tout ce qui seroit appliqué dessus offriroit à la méchanceté et à l'impiété un sujet de cupidité qui, dans des tems de troubles, pourroit amener l'idée de sa destruction " (souligné par moi). 
monument que sa valeur liturgique explicite. Cet aspect perd assurément toute pertinence dans le cas des édifices privés, et l'on peut se demander si, d'aventure, ce ne serait pas la distinction des deux classes, publique et privée, qui aurait motivé l'émergence de la catégorie du monumental.

\section{COMPOSER ENTRE PUBLIC ET PRIVÉ}

C'est, précisément, du moment que l'on est en mesure de repérer des formes intermédiaires entre les produits des commandes publiques et ceux des privées que se légitime une monumentalité de transition. Le thème est explicité en 1860 par César Daly ; il énumère alors des genres d' " édifices qui forment un lien de transition entre architecture publique et architecture privée »; ils doivent être regardés « comme des sortes de dépendances de ce grand théâtre où se développe la vie publique ${ }^{26}$. L'intuition du publiciste anticipe, en l'espèce, sur les dispositifs réglementaires de 1884 et de 1902 qui autoriseront effectivement des initiatives privées de constructions ostentatoires. En dernière analyse, le faste motive la distinction et ce n'est pas sans raison que Daly fondait sa réflexion sur les caractères de l' " inégalité moderne [...] née de la richesse $"{ }^{27}$. Les signes du pouvoir se partageront désormais entre l'autorité politique et l'autonomie des fortunes, au gré de dérogations qui tendent à privatiser certaines formes de la monumentalité. L'aspiration à mimer le faste officiel a modelé la physionomie des métropoles, en dépit des obstacles à renverser ou à contourner qu'oppose, à chaque époque édilitaire, la rigidité des gabarits réglementaires.

Dès les premiers temps de la III $^{e}$ République, en matière de monumentalité, ante litteram, la distinction du public et du privé cesse d'être impérieuse ; à preuve, le Dictionnaire de l'Académie qui, dans sa

26. La finance fascine évidemment César Daly ; les riches demeures qui font transition relèvent d'une échelle de moralité dans le théâtre social; cf. C. DALY, Architecture privée au $X I X$ siècle, Paris, 1860, p. 12 : « Moralement, par le rang et les fonctions de ceux qui les occupent, par les grandes réceptions qui s'y font, ces demeures peuvent être considérées comme... " Mais, timidement, cent ans auparavant, J.-F. BLONDEL, in L'Architecture françoise, Paris, 1754, t. III, p. 1-3, avait donné la "Description " d'une « Maison particulière [...] sise rue des Mauvaises Paroles, appartenant à M. Guillot, Intendant des Turcies et Levées ", s'excusant d'avoir " inséré cette Maison Bourgeoise " dans un Recueil des plus beaux Édifices de la capitale, en vertu de ce qu'un " Architecte doit sçavoir descendre dans le détail des plus petits Bâtimens, comme il doit sçavoir élever son imagination lorsqu'il s'agit du projet de la demeure d'un Prince, d'une Tête Couronnée, ou lorsqu'il est question d'un Édifice public "; cette ingérence du trivial se justifie encore de ce que « rien n'est plus difficile que de produire de l'excellent dans une maison de peu d'importance ».

27. Architecture privée..., op. cit. supra n. 26, p. 14. 
$7^{e}$ édition, en 1879, réunit sous la même dénomination de Monument " certains édifices publics ou particuliers qui imposent par leur grandeur ou par leur ancienneté ". L'idée d'imposer est le pivot de la définition. Il est clair que l'ancienneté, où s'absorbe l'intérêt historique, est désormais, en ce siècle d'histoire, une valeur indiscutée ${ }^{28}$. La grandeur, en revanche, est matière questionnable ; elle ne peut être entendue, dans cette définition, que comme une " grandeur qui en impose "; partant, ses bornes, toutes subjectives, sont sujettes à variations, de même que varient les représentations qui déterminent les modèles où s'inscrit le caractère monumental des métropoles.

\section{LA MONUMENTALITÉ À L'ÉPREUVE DES MATÉRIAUX}

En chaque lieu, la monumentalité implicite tient d'abord à la classification sociale des individus qui ont la charge de définir les normes du monumental et d'ordonner les moyens d'y parvenir ; ce sont rarement les mêmes. De plus, l'intérêt pour cette question d'art se définit à proportion des marchés qu'elle détermine, ou du moins de son influence sur la passation des marchés. On ne s'étonnera pas que le caractère monumental ait pu être un thème de l'incessante rivalité entre ingénieurs et architectes sur les frontières de leurs proches compétences. En mainte circonstance, la contrariété de leur argumentation fut une matrice de l'idée de monumentalité ; mais cette contrariété ne fut point statique ; elle a engendré des chassés-croisés polémiques, les partis justifiant leurs propositions dans le reflet des habiletés complémentaires. Plus précisément, la rencontre du faire-savoir et du savoir-faire se dialectise chaque fois que l'aptitude à mettre en œuvre des matériaux nouveaux est contrainte de se faire reconnaître selon des critères esthétiques.

Lorsque Navier, en 1827, projette un pont métallique devant les Invalides, il proclame bien haut que « les constructions en fer, si l'on y trouve la grandeur et la simplicité des formes, peuvent aussi bien que les édifices de pierre mériter le nom de monument $"{ }^{29}$. Dans la même veine, Reynaud, autre polytechnicien, réclame que les bâtiments faits de « matériaux jusqu'alors inusités » et présentant des « dispositions sans précédents" s'empreignent d'un authentique « caractère

28. Cf. A. Riegl, op. cit. supra n. 16, éd. Jacques Boulet, p. 42.

29. Claude-Louis Navier, De l'entreprise du Pont des Invalides, Paris, 1827, p. 4 (souligné par moi). 
monumental $\gg{ }^{30}$. Cette revendication trouve sa parade dans l'affirmation de la primauté du génie que protège le postulat d'unité des arts. Faut-il un exemple, je citerai la bravoure de Baltard, lorsqu'il annonce, en 1819, que "les monuments sont à l'architecture, ce que le genre épique est à la littérature $"{ }^{31}$. Vingt ans après, on le trouve encore plus irrité contre les ingénieurs dont les vues « étrangères à toute pensée monumentale " ne produisent que des « ouvrages grossières et à demi-barbares ${ }^{32}$. Il est vrai qu'à ce ton d'hostilité hargneuse succéderont des antagonismes plus subtils et des mancuvres d'influence plus efficaces. La célébration du don de l'art se perpétuera, certes, comme une incantation distinctive, mais les attitudes à l'égard de grands programmes incorporant des « matériaux inusités " seront diversement mitigées selon la position des acteurs dans le champ des pouvoirs de décision. De par sa notoriété et en raison du poids de ses avis dans les Commissions, Guadet pourra ainsi réclamer, sans ridicule, à la fin des années 1870 , que la monumentalité publique se réalise dans la seule pierre ${ }^{33}$. Cette fixation trouvait sa claire répartie dans le modernisme qu'exalte Boileau, architecte adepte de la construction métallique. Dès 1871 , il veut que l'on «applique » l'aluminium, à peine isolé, aux constructions, tandis que le recours au fer suffit à « achever la révolution qui s'accomplit dans l'architecture $\gg{ }^{34}$; et en 1886 , encore, impatient de l'éclectisme ambiant, il vante tapageusement l'" art de produire des impressions esthétiques au moyen de la science constructive la plus avancée $"{ }^{35}$. Nul doute qu'à ses yeux

30. Cf. Léonce REYNAUD, Traité d'architecture, Paris, $2^{e}$ éd. 1860, t. II, p. 459-460 : à propos de ces « édifices d'une nature toute spéciale » que sont « les grandes gares ». La même opinion se trouve dans C. DALY, Revue générale de l'architecture et des travaux publics, XIX, 1861, col. 78 : il qualifie la « gare de chemin de fer » de « plus haute expression monumentale et artistique du génie industriel et commercial... ". Cf. également, J.-J. Ampere, Promenades en Amérique, Paris, 1855, p. 358.

31. L.-P. BALTARD, Discours d'ouverture du cours de théorie de l'architecture, Paris, s.d. [1819], p. 14.

32. ID., Introduction..., op. cit. supra n. 17, p. 18.

33. Cf. "Conférence sur le Nouvel hôtel des Postes ", extrait des Mémoires de la Société des ingénieurs civils, mai 1886 ; Jules GUADET y proclame sans ambage que " la pierre seule est monumentale " .

34. Cf. Louis-Charles BolleAu, Le Fer, principe élémentaire constructif de la nouvelle architecture, Paris, 1871, p. 26. Mais son intervention est déjà tardive, eu égard aux prises de position d'Hector HOREAU; cf., p. ex., ses commentaires sous le titre « Halles centrales ", Revue générale de l'architecture et des travaux publics, 1857, col. 104, où il reconnaît aux pavillons de Baltard « un air suffisamment monumental, sans perdre le caractère de construction légère qui doit appartenir à un marché... " "

35. L.-C. Bolleau, Histoire critique de l'invention en architecture, Paris, 1886. L'idée figurait déjà dans son ouvrage sur Le Fer..., op. cit. supra, p. 28, empreinte de rationalité technologique, lorsqu'il prétendait que « le vrai mérite dans l'art de bâtir [...] consiste à obtenir les plus grands effets avec le moins d'efforts possibles ». 
le métal n'aille au monumental; il rejoint systématiquement les arguments que prétendait Navier, pour faire agréer ses projets dans un temps imbu des stéréotypes de la solidité apparente. Mais une chose est la présentation casuelle ou systématique d'un mode de construction que l'on crédite de la capacité d'atteindre au monumental ; une autre l'exploitation de sa mise en œuvre. Et c'est sur ce sujet que se déporte la concurrence entre architectes et ingénieurs dont la littérature spéciale de la seconde moitié du siècle nous a laissé maintes traces où la suffisance d'un esprit concordataire révèle l'ampleur du malaise. Il suffit de relire Garnier prenant acte de la "distinction assez moderne " de la " classification des ingénieurs et des architectes ", pour aussitôt questionner sur la " juste démarcation des compétences et des programmes " ${ }^{36}$, alors qu'Anatole de Baudot avait, en 1864, diagnostiqué les motifs de la faveur dont jouissent les ingénieurs, indemnes de "parti pris ", appliqués à " remplir rigoureusement le programme qui leur est donné $"{ }^{37}$. C'était implicitement reconnaître que l'obligation de moyens se sépare de l'obligation de résultats et, en définitive, la question du monumental, si troublée qu'elle fût par le bruit des corps hostiles, demeurait affaire de conception d'ensemble et de jugement esthétique. Le Dictionnaire de l'Académie nous ramène à une juste appréciation ; le seul problème digne d'intérêt, tel qu'il résulte de la définition de 1879 , consiste à retracer la genèse des conditions qui assignent à chaque état social les dimensions et les traits de la « grandeur qui impose ».

\section{MASSE ET MONUMENTALITÉ}

On peut lire dans Alain que "la solidité et on dirait presque la sincérité des monuments éclate encore mieux par leur grandeur et par leur masse ; c'est pourquoi on peut aller jusqu'à dire que la beauté architecturale dépend beaucoup de la masse dressée "; à l'appui de quoi il cite le cas des Pyramides dont la masse fait « presque

36. Charles Garnier, $A$ travers les arts, Paris, 1869, p. 72-73. p. 4.

37. Anatole DE BAudot, Réorganisation de l'École des beaux-arts..., Paris, 1864, 
la seule beauté ${ }^{38}$. L'exemple est pertinent, mais rebattu ; il a nourri les doutes esthétiques deux siècles durant, dans un ramage de doctrine dont le beau et le sublime, la nature et l'artifice donnaient les repères instables. Delacroix confie en 1852 à son Journal que «Tout ce qui est grand produit à peu près la même sensation ". L'« effet sublime " de la mer est à ses yeux " celui d'une énorme quantité d'eau "; l'ombre qui agrandit le soir la masse d'une église est cause qu'il « éprouve la sensation du sublime ${ }^{39}$. Trente ans auparavant, un commentaire anonyme de " l'illusion dans les Beaux-Arts " assurait que l'architecture est « sans expression autre que celle qui naît de la grandeur des masses [et] qui ne produit que l'étonnement dont nous sommes toujours saisis à l'aspect des hautes montagnes ${ }^{40}$. Le parallèle est légitime, mais peu instructif. Ce qui mérite plus d'attention, c'est la manière dont on peut envisager l'économie esthétique de la grandeur, en y comprenant les ménages de l'illusion. Après les pyramides d'Égypte, la basilique vaticane entre dans le feu des jugements que met en cause le regain d'attention pour les temples gothiques. On connaît le dépit de Laugier qui regardait " comme un très grand défaut qu'un édifice pour lequel on a fait de si grands frais ne produise pas un effet extraordinaire $"{ }^{41}$. À cette humeur répond le subtil Burke, l'un des premiers qui fut à saisir la complexité des rapports entre mesures réelles et apparentes et à indiquer les ruses de l'art dans la recherche de l'optimum de grandeur, à la faveur de quelque "généreuse imposture "; ce qui lui fit dire qu' « aucun ouvrage de l'art n'est grand qu'autant qu'il trompe $"{ }^{42}$. Ce qu'énoncera, en d'autres termes, Emiland Gauthey en prétendant qu'« un

38. Passage tiré du Système des beaux-arts, Paris, 1920, rééd. in Les Arts et les Dieux, Paris, 1958, p. 342. Cf., déjà en 1801, LusSaulT, Journal des bâtiments civils, des sciences et des arts, 3 germinal, an IX, p. 6, qui professait une admiration analogue, c'est-à-dire nuancée : " un édifice très-grand et majestueux, mais dont l'aspect est uniforme (tel est celui des pyramides d'Égypte), saisit d'abord, et excite au premier coup d'œil un ravissement irrésistible et inexplicable. L'âme étonnée ne conçoit pas comment ce phénomène de l'art peut lui plaire, même en contrariant les formes et les intentions de la nature ; mais bientôt ne trouvant dans l'objet de sa contemplation aucun aliment à sa curiosité, rien qui prolonge sa surprise et entretienne son admiration, l'excès de l'uniformité la fatigue... " Cependant, la pyramide demeure le schéma convenu de la majesté monumentale : cf. Charles PerCIER, Louis FonTAINE, Résidences de souverains, Paris, 1833, p. 342 : un « édifice est majestueux lorsque par son immensité il domine tout ce qui l'environne ", étant de « forme régulière ", doté, en outre d'une " magnificence et d'un éclat " mesurés.

39. À la date du dimanche 12 septembre, éd. Paul FLAT, Paris, 1893, p. 117 et 118 . Même ressort dans ce passage du livre d'Ann RADCLIFFE, Mystère d'Udolphe, trad. de Victorine DE Chastanay, Paris, an V, t. II, p. 112 : « Isolé, vaste et massif [le château] sembloit dominer la contrée ; plus la nuit devenoit obscure, plus ses tours isolées paroissent imposantes. "

40. Cf. Annales françaises des arts, des sciences et des lettres, t. XI, 1822, p. 166.

41. Abbé Laugier, Observations sur l'architecture, Paris, 1765, p. 55.

42. Dans la traduction de LAGENTIE de LAVAISSE de la Recherche philosophique sur l'origine de nos idées du Sublime et du Beau, Paris, an XI, p. 137. 
édifice est presque toujours beau lorsqu'il est grand et qu'il ne choque point la vraisemblance ", mais que l'" on se plaît davantage à examiner celui qui ne paroîtra grand que par l'effet des illusions optiques $\${ }^{43}$. Ce genre de propos témoigne du soin de quelques forts esprits appliqués à établir des relations entre échelle architecturale et induction du monumental ${ }^{44}$. La question est d'une approche malaisée ; les termes n'en sont pas fixés et nul invariant anthropologique ne peut être invoqué pour repérer la transition historique des objets en cause. Sans doute, peut-on regarder du côté de l'idée d'ensemble, au sens esthétique, en vue de formuler une ébauche de ces relations. Quatremère de Quincy s'y est risqué ; à ses yeux, "l'ensemble le plus essentiel est celui des proportions et des masses » qui ne se réduit pas à de claires raisons ; certaines des dimensions déterminantes « dépendent de la position de l'édifice, de son point de vue et des accessoires qui l'accompagnent, et que le goût seul de l'artiste peut fixer $"{ }^{45}$. On devrait s'excuser de ranimer de pareilles évidences, mais elles ont un mérite, celui de rappeler que le caractère transitoire de la monumentalité est relatif à un environnement proche. Lorsque Chastenet déplore à la Chambre, en 1909, le caractère erratique de l'habitat parisien qui le rapproche des villes américaines, il insiste sur « la nécessité de subordonner les édifices privés aux monuments publics ${ }^{46}$. Le monumental, on l'a compris, est moins une affaire de volumétrie que de marquage et d'isolement ostentatoire des lieux de pouvoir. Inversement, dans la mesure où les villes se texturent et s'enrichissent, la surrection monumentale des édifices privés devient un problème que perpétue le désir d'ostentation. Pour les édiles, le problème consiste à déterminer les limites dans lesquelles peuvent être tolérées mais parfois, encouragées, des diversités d'aspects sous la loi qui

43. Cf. « Mémoire sur les règles de l'architecture », Ms. École des ponts et chaussées, $1826 / 4^{\circ}, f^{\circ} 193-194$. Dirigée en sens contraire, la louange de la généreuse imposture fut faite par Fénelon sur le sujet des édifices des Grecs; cf. FÉnELON, Lettres sur les occupations de l'Académie, 1714, cité in Chefs d'ceuvre littéraires de Fénelon, Paris, 1839, p. 720 : «Les proportions [y] sont si justes que rien ne paroît fort grand quoique tout le soit."

44. L'idée en est formulée dans cette proposition de P. F. BIANCHI, in l'Istituzione pratica dell'architettura civile, Milan, 1768, p. 102 : « La Magnificenza può essere ugualmente nel grande che nel piccolo... " L'un des procédés corrélatifs, en faveur chez les néo-classiques, est de veiller à la « parcimonie dans la division des parties [...] seul moyen de donner aux édifices ce caractère imposant qui frappe, qui étonne et qui commande l'admiration ", cf. G. A. BoRGNIS, Traité élémentaire de construction appliqué à l'architecture civile, Paris, 1823, p. 406.

45. Cf. Encyclopédie méthodique, Architecture, Paris, an IX, t. II, V०, " Ensemble ", p. 338 .

46. Discours du 24 juin 1909, cité in C. Lortsch, La Beauté de Paris et la loi, Paris, 1913 , p. 14. 
régit approximativement l'ensemble des parties de l'organisme urbain. En un sens que désigne la suite des dérogations, on retrouve, à l'échelle des métropoles, une posture analogue à celle qui détermine les luttes de domination à l'intérieur de groupes primitifs. Cependant, les urbanistes européens ont eu le génie, durant les dernières décennies, de substituer à l'ancienne "logique " de la subordination, une nouvelle manière d'aborder la monumentalité ; sous deux chefs dissemblables, l'« intégration " des monuments anciens dans le tissu urbain renouvelé, et la désintégration des procédés de la monumentalité dans la dérision du "post-moderne $"{ }^{47}$.

Jacques GUILLERME, C.N.R.S., Paris.

47. Cf. G. R. Collins, "Monumentality : A Critical Matter in Modern Architecture ", The Harvard Architecture Review, Spring 1984, passim. Également, Giulio DubBINI, " Progetto, locus, monumento ", in G. Braghiari, \& coll., Progetti Veneziani, Milan, 1985. 